improved technology and experimental techniques. Data from the ion probe were presented and in one study of a fine-grained iron meteorite a scanning device on a transmission electron microscope was used to obtain high resolution spot analyses across the interface between $\alpha$ (low- $\mathrm{Ni}$ ) and $\gamma$ (high-Ni) metal. J. I. Goldstein and coworkers at Lehigh University, Pennsylvania intend to apply this technique to experimentally produced structures in an attempt to measure diffusion rates at low temperatures (below about $600^{\circ} \mathrm{C}$ ).

\section{More light on enzyme control}

\section{from C. J. Lamb and R. K. Dudley}

IT has been said in these columns that 'Plant molecular biology is not dead, but is merely awaiting the attention of competent and dedicated biochemists', (News and Views 254, 13; 1975). A recent illustration of the potential contribution that biochemical studies can make to the understanding of plant biology is given by the work of Klaus Hahlbrock and colleagues at the University of Freiburg on the photocontrol of phenylalanine ammonia-lyase (PAL) levels in parsley cell cultures. By looking at the production of PAL in cellfree systems they have been able to show that the increase in PAL production observed after illumination is due to an increase in translatable mRNA and not to activation of previously synthesised enzyme.

PAL catalyses the deamination of L-phenylalanine to trans-cinnamic acid, the first step in the biosynthesis of plant compounds based on the phenylpropane unit and including lignins, flavonoids and coumarins. Its activity increases greatly following illumination of various plant tissues and the enzyme has proved popular for studying the photocontrol of gene expression in higher plants. Unfortunately, many of these investigations have relied on the effects of the antimetabolites actinomycin D and cycloheximide, both of which have many different effects in higher plants. Furthermore, densitylabelling studies of the phytochromemediated increase in PAL activity in mustard seedlings have produced conflicting results and there is considerable controversy as to whether in this case enhancement of PAL activity is caused by activation of previously synthesised Chris Lamb is in the School of Botany at
the University of Oxford and Keith Dudley is in the Department of Biochemistry at the University of Cambridge. protein or by increased de novo synthesis. Hahlbrock's contribution has been to complement antimetabolite and in vivo labelling experiments with a careful study of the synthesis of PAL in cell-free systems. The results obtained to date suggest that this approach will give insight into a number of fundamental problems in plant biology.

Early work (reviewed by Hahlbrock Physiol. Veg. 14, 207; 1976) demonstrated that on irradiation with blue or ultraviolet light, previously dark-grown parsley cell suspension cultures accumulate a number of flavonoids concurrent with a large increase and subsequent decrease in the extractable activity of PAL. These responses are red/far-red reversible, but neither red nor far-red light has an effect without ultraviolet preirradiation, suggesting that the initial responses are not phytochromemediated. The ability of light to enhance PAL activity declines progressively, and after a few hours illumination further irradiation has no stimulatory effect. In vivo studies with actinomycin D and cycloheximide and labelling with ${ }^{35} \mathrm{~S}$-methionine and ${ }^{15} \mathrm{~N}$ ammonia indicated that PAL was synthesised de novo following illumination. The light-mediated changes in the apparent rate of synthesis of the enzyme were calculated from the timecourse of changes in extractable PAL activity under various programmes of illumination, and the results interpreted as reflecting short-term light-mediated increases in the concentration of specific mRNA available for translation.

This hypothesis has now been investigated directly by study of the biosynthesis of PAL in cell-free systems. In vitro protein synthesis with polyribosomes from parsley cell cultures was relatively low with a homologous system, but an effective heterologous system was established by supplementing the parsley ribosomes with an extract from wheat germ (Schröder \& Hahlbrock Biochim. biophys. Acta 366, $454 ; 1974)$. The rate of synthesis of PAL was determined by incorporation of ${ }^{35} \mathrm{~S}$-methionine into protein precipitable by a rabbit antiserum prepared against the purified enzyme (Schröder, Betz \& Hahlbrock Eur J. Biochem. 67, 527; 1976). Analysis of the immunoprecipitate by disc gel electrophoresis in the presence of dodecylsulphate revealed small amounts of the complete enzyme subunit (molecular weight 83,000 ) and relatively large amounts of shorter peptides which were also immunologically characteristic of the enzyme; an extra check by tryptic fingerprinting would have been desirable.

The capacity for PAL synthesis was much greater with polyribosomes from irradiated cells compared with those from dark-grown cells and the observed changes in the rate of PAL synthesis closely corresponded with those previously calculated from changes in the level of extractable PAL activity. The possibility that the increased capacity for PAL synthesis reflects changes in the amount or activity of ribosomebound factors rather than changes in the level of translatable mRNA has been ruled out by extracting the RNA from parsley polyribosome preparations with chloroform/phenol and using this extract as a message in a rabbit reticulocyte lysate (Ragg, Schröder \& Hahlbrock Biochim. biophys. Acta 474, 226, 1977). Synthesis of PAL could only be detected using RNA from lighttreated cultures. This is the first report that a mammalian cell-free system will accept a plant message for translation, and is especially interesting since PAL is an enzyme of plant secondary metabolism and is not found in animals.

These studies demonstrate an increase in the level of translatable PAL mRNA in response to illumination, but is this a result of more message being produced, or less being destroyed, or to pre-existing message being made available for translation, or indeed to a combination of these possibilities? To answer this will require purification of the PAL mRNA, a daunting task as the message is likely to be only a tiny proportion of the total RNA extracted. However, enhancement of the level of PAL activity is accompanied by concurrent increases in the subsequent enzymes of the pathway and using Hahlbrock's approach it should be possible to analyse the mechanisms whereby coordinated gene expression in higher plants is achieved in response to environmental stimuli. Of immediate interest is the phytochrome-mediated stimulation of phenylpropanoid biosynthesis in intact plants. The regulatory mechanisms may well differ from those observed with cell cultures undergoing rapid cell division and nucleic acid biosynthesis.

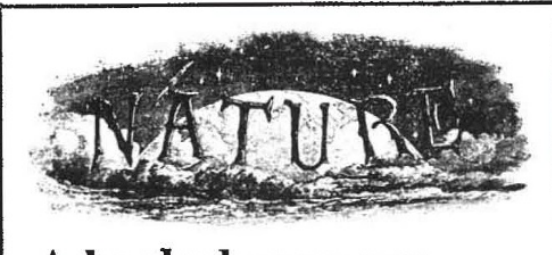

\section{A hundred years ago}

THE Frigorifique, fitted up for the transportation of meat on the Tellier system with methylic acid, has arrived at Havre, from Brazil, with its cargo in an excellent state of preservation. It is stated that a banquet of the meat will be served during the forthcoming session of the French Association at Havre.

From Nature 16, 23 August, 351; 1877. 